\title{
Novel Control Strategy for Maximum-Boiling Extractive Distillation Systems: Acetone/Chloroform Separation
}

\section{Supporting Information}

\author{
Meng-Lin Tsai, Yen-Hsiang Wang and I-Lung Chien* \\ Department of Chemical Engineering \\ National Taiwan University \\ Taipei 10617, Taiwan
}

April 10, 2020 
This text file is the supporting information for the paper "Novel Control Strategy for Maximum-Boiling Extractive Distillation Systems: Acetone/Chloroform Separation"

Figuer S1. Detailed results of SVD analysis for the extractive distillation column

Figure S2. Calculation results of $Z_{i}$

Figure S3. Detailed results of RGA analysis

Figure S4. Detail results of NRG analysis and the calculation of row sum of $\Lambda^{N}$ 
Figuer S1. Detailed results of SVD analysis for the extractive distillation column

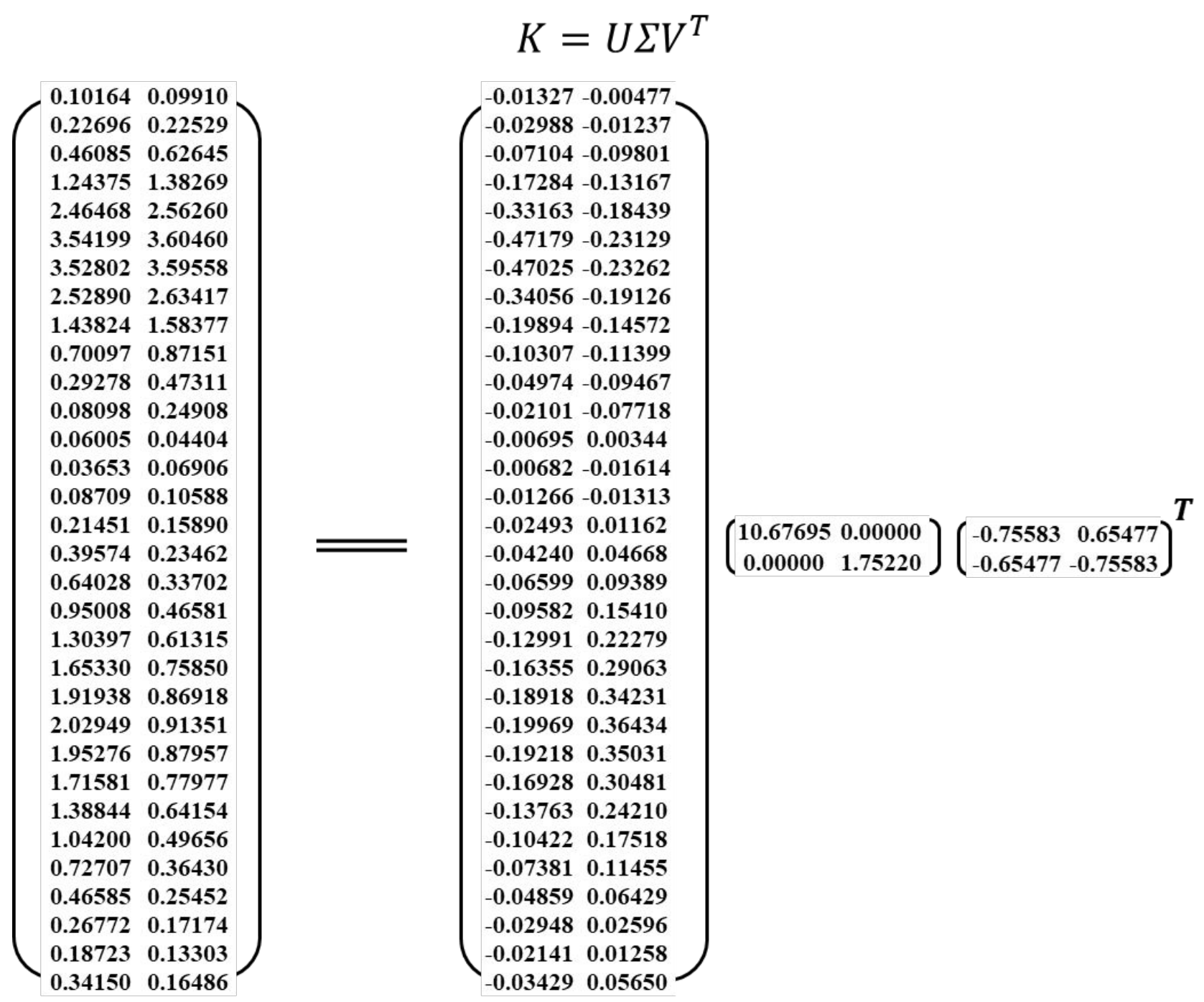


Figure S2. Calculation results of $Z_{i}$

$$
Z_{i}=\left|U_{1 i}\right|-\left|U_{2 i}\right|
$$

$\left(\begin{array}{c}0.00851 \\ 0.01752 \\ -0.02697 \\ 0.04117 \\ 0.14724 \\ 0.24050 \\ 0.23763 \\ 0.14931 \\ 0.05322 \\ -0.01092 \\ -0.04493 \\ -0.05618 \\ 0.00351 \\ -0.00932 \\ -0.00047 \\ 0.01331 \\ -0.00427 \\ -0.02789 \\ -0.05827 \\ -0.09288 \\ -0.12708 \\ -0.15313 \\ -0.16465 \\ -0.15813 \\ -0.13553 \\ -0.10447 \\ -0.07097 \\ -0.04074 \\ -0.01571 \\ 0.00353 \\ 0.00883 \\ -0.02221\end{array}\right)$

$$
\begin{array}{|l|l}
-0.01327 \\
-0.02988 \\
-0.07104 \\
-0.17284 \\
-0.33163 \\
-0.47179 \\
-0.47025 \\
-0.34056 \\
-0.19894 \\
-0.10307 \\
-0.04974 \\
-0.02101 \\
-0.00695 \\
-0.00682 \\
-0.01266 \\
-0.02493 \\
-0.04240 \\
-0.06599 \\
-0.09582 \\
-0.12991 \\
-0.16355 \\
-0.18918 \\
-0.19969 \\
-0.19218 \\
-0.16928 \\
-0.13763 \\
-0.10422 \\
-0.07381 \\
-0.04859 \\
-0.02948 \\
-0.02141 \\
-0.03429
\end{array} \mid
$$

$-0.00477$

$-0.01237$

$-0.09801$

$-0.13167$

$-0.18439$

$-0.23129$

$-0.23262$

$-0.19126$

$-\mathbf{0 . 1 4 5 7 2}$

$-0.11399$

$-0.09467$

$-0.07718$

0.00344

$-0.01614$

$-0.01313$

0.01162

0.04668

0.09389

0.15410

0.22279

0.29063

$\mathbf{0 . 3 4 2 3 1}$

0.36434

0.35031

0.30481

0.24210

0.17518

0.11455

0.06429

0.02596

0.01258

0.05650

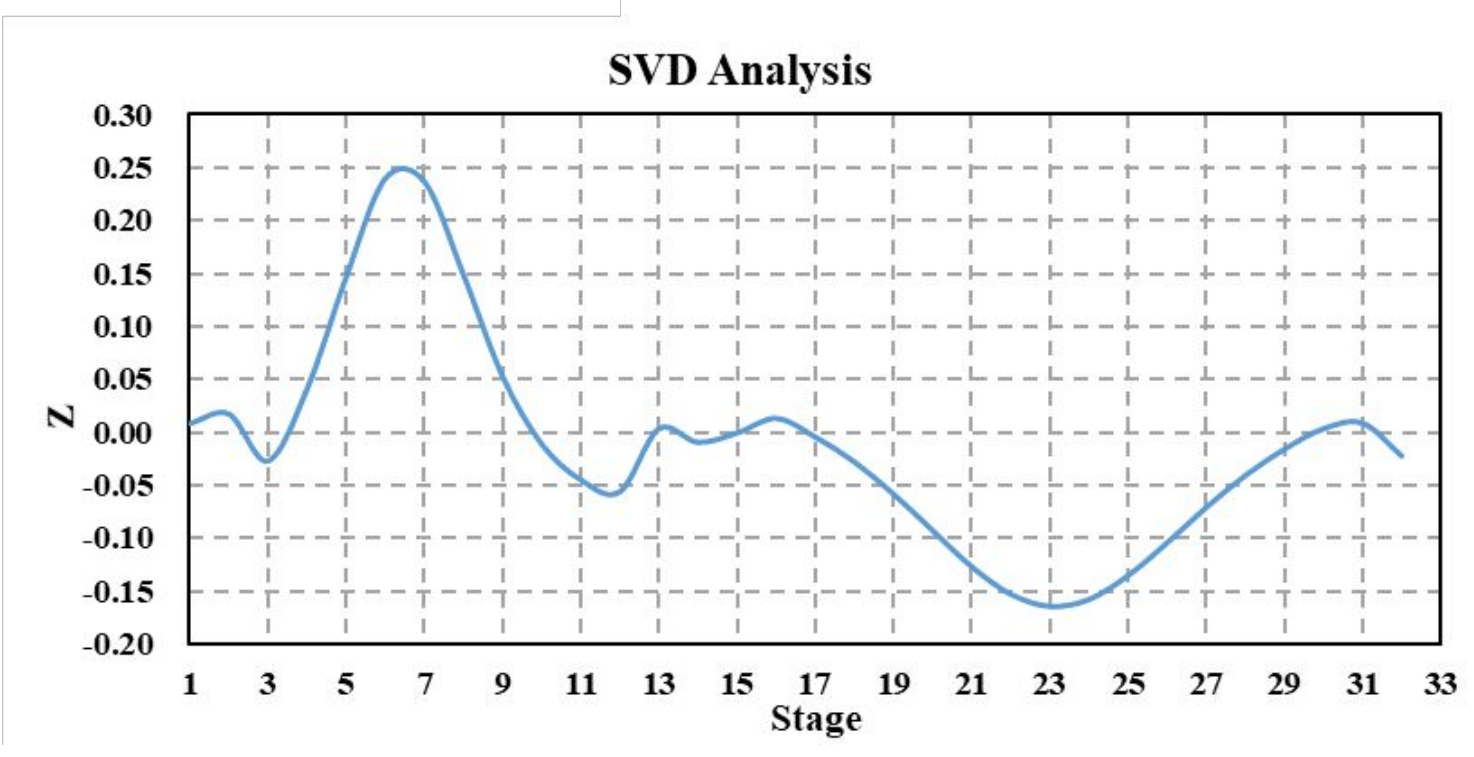


Figure S3. Detailed results of RGA analysis

$$
\begin{aligned}
& \Lambda=K \otimes\left(K^{-1}\right)^{T} \\
& \left(\begin{array}{cc}
-0.79103 & 1.79103 \\
1.79103 & -0.79103
\end{array}\right)=\left(\begin{array}{ll}
3.52802 & 3.59558 \\
2.02949 & 0.91351
\end{array}\right) \otimes\left(\begin{array}{cc}
-0.22421 & 0.49812 \\
0.88250 & -0.86592
\end{array}\right) \\
& \Lambda=\left[\begin{array}{cc}
\mathrm{QR} 1 / \mathrm{FF} & \mathrm{RF} 1 / \mathrm{FF} \\
-0.79103 & \mathbf{1 . 7 9 1 0 3} \\
\mathbf{1 . 7 9 1 0 3} & -0.79103
\end{array}\right] \begin{array}{l}
7 \\
23
\end{array}
\end{aligned}
$$


Figure S4. Detail results of NRG analysis and the calculation of row sum of $\Lambda^{N}$

$$
\Lambda^{N}=K \otimes\left(K^{+}\right)^{T}
$$

$\left(\begin{array}{cc}-0.00009 & 0.00028 \\ -0.00057 & 0.00161 \\ -0.01456 & 0.02922 \\ -0.04598 & 0.09319 \\ -0.11196 & 0.25594 \\ -0.18783 & 0.46392 \\ -0.18923 & 0.46448 \\ -0.11977 & 0.27234 \\ -0.05806 & 0.11888 \\ -0.02474 & 0.04836 \\ -0.00933 & 0.02076 \\ -0.00222 & 0.00861 \\ 0.00011 & -0.00005 \\ -0.00020 & 0.00051 \\ -0.00035 & 0.00068 \\ 0.00131 & -0.00055 \\ 0.00809 & -0.00411 \\ 0.02545 & -0.01229 \\ 0.06115 & -0.02823 \\ 0.12055 & -0.05404 \\ 0.19870 & -0.08748 \\ 0.27122 & -0.11826 \\ 0.30500 & -0.13238 \\ 0.28219 & -0.12254 \\ 0.21600 & -0.09443 \\ 0.13914 & -0.06158 \\ 0.07590 & -0.03435 \\ 0.03492 & -0.01635 \\ 0.01279 & -0.00630 \\ 0.00316 & -0.00161 \\ 0.00116 & -0.00055 \\ 0.00804 & -0.00367\end{array}\right)$
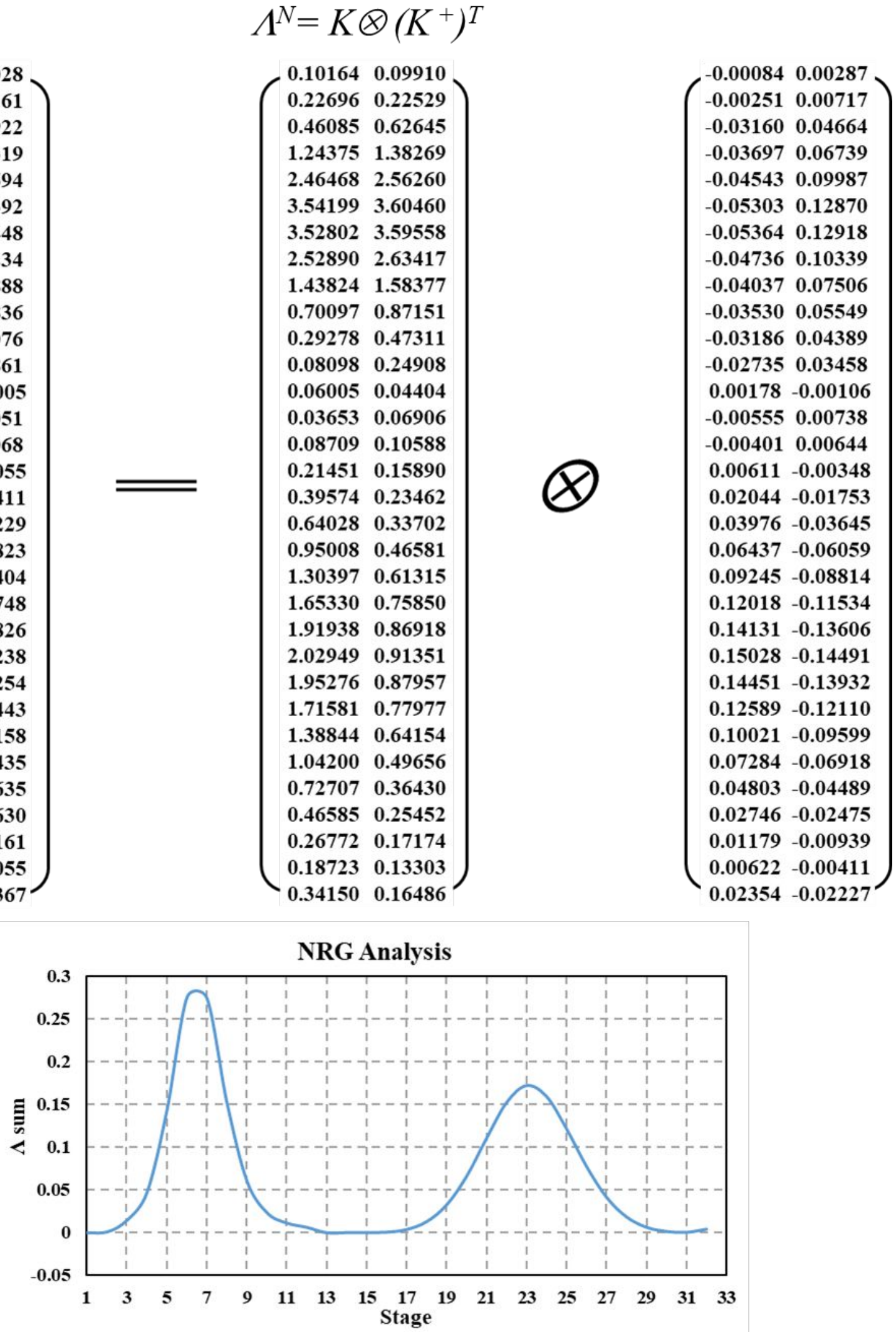